TRANSACTIONS OF THE

AMERICAN MATHEMATICAL SOCIETY

Volume 362, Number 11, November 2010, Pages 6065-6077

S 0002-9947(2010)05068-8

Article electronically published on July 7, 2010

\title{
SOME NEW CLASSES OF COMPLEX SYMMETRIC OPERATORS
}

\author{
STEPHAN RAMON GARCIA AND WARREN R. WOGEN
}

\begin{abstract}
We say that an operator $T \in B(\mathcal{H})$ is complex symmetric if there exists a conjugate-linear, isometric involution $C: \mathcal{H} \rightarrow \mathcal{H}$ so that $T=C T^{*} C$. We prove that binormal operators, operators that are algebraic of degree two (including all idempotents), and large classes of rank-one perturbations of normal operators are complex symmetric. From an abstract viewpoint, these results explain why the compressed shift and Volterra integration operator are complex symmetric. Finally, we attempt to describe all complex symmetric partial isometries, obtaining the sharpest possible statement given only the data $\left(\operatorname{dim} \operatorname{ker} T, \operatorname{dim} \operatorname{ker} T^{*}\right)$.
\end{abstract}

\section{INTRODUCTION}

Throughout this paper, $\mathcal{H}$ will denote a separable complex Hilbert space and all operators considered will be bounded. We first require a few preliminary definitions:

Definition. A conjugation is a conjugate-linear operator $C: \mathcal{H} \rightarrow \mathcal{H}$ that is both involutive $\left(C^{2}=I\right)$ and isometric. We say that a bounded linear operator $T \in B(\mathcal{H})$ is $C$-symmetric if $T=C T^{*} C$ and complex symmetric if there exists a conjugation $C$ with respect to which $T$ is $C$-symmetric.

It is not hard to see that $T$ is a complex symmetric operator if and only if $T$ is unitarily equivalent to a symmetric matrix with complex entries, regarded as an operator acting on an $l^{2}$-space of the appropriate dimension (see [9, Sect. 2.4] or [7. Prop. 2]).

The class of complex symmetric operators includes all normal operators, operators defined by Hankel matrices, compressed Toeplitz operators (including finite Toeplitz matrices and the compressed shift), and the Volterra integration operator. We refer the reader to [7, 8] (or [9] for a more expository pace) for further details. Other recent articles concerning complex symmetric operators include [3, 11.

In this paper, we exhibit several additional classes of complex symmetric operators. In particular, we establish that

(i) All binormal operators are complex symmetric (Theorem 1) and that $n$ normal operators that are not complex symmetric exist for each $n \geq 3$ (Example 1).

Received by the editors March 17, 2009.

2000 Mathematics Subject Classification. Primary 47B99.

Key words and phrases. Complex symmetric operator, normal operator, binormal operator, nilpotent operator, idempotent, partial isometry.

The first author was partially supported by National Science Foundation Grant DMS-0638789. 
(ii) Operators that are algebraic of degree two are complex symmetric (Theorem 22). This includes all idempotents and all operators that are nilpotent of order 2 .

(iii) Large classes of rank-one perturbations of normal operators are complex symmetric (Theorem 3). On abstract grounds, this explains why the compressed shift operator (Example 2) and Volterra integration operator (Example 3) are complex symmetric.

(iv) We attempt to describe all complex symmetric partial isometries, obtaining the sharpest possible statement (Theorem 4) given only the data $\left(\operatorname{dim} \operatorname{ker} T, \operatorname{dim} \operatorname{ker} T^{*}\right)$.

\section{BinORMAL OPERATORS AND $n$-NORMAL OPERATORS}

Definition. An operator $T \in B(\mathcal{H})$ is called binormal if $T$ is unitarily equivalent to an operator of the form

$$
\left(\begin{array}{ll}
N_{11} & N_{12} \\
N_{21} & N_{22}
\end{array}\right)
$$

where the entries $N_{i j}$ are commuting normal operators. More generally, we say that $T$ is n-normal if $T$ is unitarily equivalent an $n \times n$ operator matrix whose entries are commuting normal operators.

Needless to say, each $n \times n$ scalar matrix trivially defines an $n$-normal operator on $\mathbb{C}^{n}$. For further information concerning binormal and $n$-normal operators, we refer the reader to [12, 15$]$.

The main theorem of this section is:

Theorem 1. If $T \in B(\mathcal{H})$ is a binormal operator, then $T$ is a complex symmetric operator. This result is sharp in the sense that if $n \geq 3$, then there exists an n-normal operator that is not a complex symmetric operator.

Proof. We focus our attention on the first statement, since the second will follow from the construction of explicit examples (see Example 1). Given an operator of the form (1), the Spectral Theorem asserts that we may assume that each $N_{i j}$ is a multiplication operator $M_{u_{i j}}$ on a Lebesgue space $L^{2}(\mu)$ where $\mu$ is a Borel measure on $\mathbb{C}$ with compact support $\Delta$ and that the corresponding symbols $u_{i j}$ belong to $L^{\infty}(\mu)$. To simplify our notation, we will henceforth identify multiplication operators $M_{u}$ with their symbols $u$.

Without loss of generality, we may further restrict our attention to operators on $L^{2}(\mu)^{(2)}$ (the two-fold inflation of $\left.L^{2}(\mu)\right)$ of the form

$$
T=\left(\begin{array}{cc}
u_{11} & u_{12} \\
0 & u_{22}
\end{array}\right),
$$

since any binormal operator is unitarily equivalent to an operator of form (2) [15, Thm. 7.20].

Let us denote by $E$ the subset of $\Delta$ upon which $u_{11}=u_{22}$ :

$$
E=\left\{z \in \Delta: u_{11}(z)=u_{22}(z) \mu \text {-a.e. }\right\} \text {. }
$$

Letting $\chi_{E}$ denote the characteristic function of $E$, we note that the subspace $\mathcal{E}_{1}=\chi_{E} L^{2}(\mu)$ and its orthogonal complement $\mathcal{E}_{2}=\chi_{\Delta \backslash E} L^{2}(\mu)$ are both reducing 
subspaces for $M_{z}: L^{2}(\mu) \rightarrow L^{2}(\mu)$, the operator of multiplication by the independent variable. In particular, their inflations $\mathcal{E}_{1}^{(2)}$ and $\mathcal{E}_{2}^{(2)}$ are both reducing subspaces for $T$, and we see that

$$
T=\left.\left.T\right|_{\mathcal{E}_{1}^{(2)}} \oplus T\right|_{\mathcal{E}_{2}^{(2)}} .
$$

Since the direct sum of complex symmetric operators is complex symmetric, we need only consider the following two special cases:

(i) $u_{11}=u_{22} \mu$-a.e.,

(ii) $u_{11} \neq u_{22} \mu$-a.e.

Case (i). Suppose that $u_{11}=u_{22} \mu$-a.e. In this case, we may write (2) as

$$
\left(\begin{array}{ll}
u & v \\
0 & u
\end{array}\right)
$$

where $u, v \in L^{\infty}(\mu)$. One can immediately verify that $T$ is $C$-symmetric with respect to the conjugation $C\left(f_{1}, f_{2}\right)=\left(\overline{f_{2}}, \overline{f_{1}}\right)$ on $L^{2}(\mu)^{(2)}$.

Case (ii). Suppose that $u_{11} \neq u_{22} \mu$-a.e. In this case, $T$ has the form

$$
\left(\begin{array}{cc}
u_{1} & v \\
0 & u_{2}
\end{array}\right)
$$

where $u_{1} \neq u_{2} \mu$-a.e. Let $F$ denote the subset of $\Delta$ upon which $v$ vanishes, and observe that $T=\left.\left.T\right|_{\mathcal{F}_{1}^{(2)}} \oplus T\right|_{\mathcal{F}_{2}^{(2)}}$, where $\mathcal{F}_{1}=\chi_{F} L^{2}(\mu)$ and $\mathcal{F}_{2}=\chi_{\Delta \backslash F} L^{2}(\mu)$. Since $v$ vanishes on $F$, it follows from (3) that $\left.T\right|_{\mathcal{F}_{1}^{(2)}}$ is normal and hence complex symmetric. On the other hand, $\left.T\right|_{\mathcal{F}_{2}^{(2)}}$ is an operator of the form (3), where $v$ is $\mu$-a.e. nonvanishing. Without loss of generality, we may therefore assume that $v$ does not vanish on a set of positive $\mu$-measure.

Since $u_{1}-u_{2}$ and $v$ are nonvanishing $\mu$-a.e., we may define a unimodular function $\gamma$ by the formula

$$
\gamma=\frac{v}{|v|} \cdot \frac{\left|u_{1}-u_{2}\right|}{\left(u_{1}-u_{2}\right)}
$$

Letting

$$
a(z)=\frac{\gamma\left|u_{1}-u_{2}\right|}{\sqrt{\left|u_{1}-u_{2}\right|^{2}+|v|^{2}}}, \quad b(z)=\frac{|v|}{\sqrt{\left|u_{1}-u_{2}\right|^{2}+|v|^{2}}},
$$

we note that the operator

$$
U=\left(\begin{array}{cc}
a & b \\
b & -\bar{a}
\end{array}\right)
$$

on $L^{2}(\mu)^{(2)}$ is unitary since $b$ is real and $|a|^{2}+|b|^{2}=1 \mu$-a.e.

Let $J f=\bar{f}$ denote the canonical conjugation on $L^{2}(\mu)$ and let $K=J^{(2)}$ denote its two-fold inflation:

$$
K=\left(\begin{array}{ll}
J & 0 \\
0 & J
\end{array}\right) .
$$

Clearly $K$ is a conjugation on $L^{2}(\mu)^{(2)}$, and a short computation shows that $U^{*}=$ $K U K$ (i.e. $U$ is a $K$-symmetric operator). 
We now claim that $C=U K$ is a conjugation on $L^{2}(\mu)^{(2)}$. Since $C$ is obviously conjugate-linear and isometric, we need only verify that $C^{2}=I$ :

$$
C^{2}=(U K)(U K)=U(K U K)=U U^{*}=I .
$$

Thus $C$ is a conjugation on $L^{2}(\mu)^{(2)}$, as claimed.

We conclude the proof by showing that $T$ is $C$-symmetric. We will do this by directly verifying that $C T^{*}=T C$. First note that

$$
\begin{aligned}
T C & =T U K \\
& =\left(\begin{array}{cc}
u_{1} & v \\
0 & u_{2}
\end{array}\right)\left(\begin{array}{cc}
a & b \\
b & -\bar{a}
\end{array}\right) K \\
& =\left(\begin{array}{cc}
a u_{1}+b v & b u_{1}-\bar{a} v \\
b u_{2} & -\bar{a} u_{2}
\end{array}\right) K .
\end{aligned}
$$

On the other hand, we also have

$$
\begin{aligned}
C T^{*} & =U K T^{*} \\
& =\left(\begin{array}{cc}
a & b \\
b & -\bar{a}
\end{array}\right) K\left(\begin{array}{cc}
\overline{u_{1}} & 0 \\
\bar{v} & \overline{u_{2}}
\end{array}\right) \\
& =\left(\begin{array}{cc}
a & b \\
b & -\bar{a}
\end{array}\right)\left(\begin{array}{cc}
u_{1} & 0 \\
v & u_{2}
\end{array}\right) K \\
& =\left(\begin{array}{cc}
a u_{1}+b v & b u_{2} \\
b u_{1}-\bar{a} v & -\bar{a} u_{2}
\end{array}\right) K .
\end{aligned}
$$

To verify the equality of (6) and (7), we need only show that $b u_{2}=b u_{1}-\bar{a} v$. However, the preceding equation follows directly from (4) and (5).

One might regard Theorem 1 as a generalization of the following well-known result (alternate proofs of which can be found in [3, Cor. 3.3], [7, Ex. 6], or [18, Cor. 3]):

Corollary 1. Every linear operator on $\mathbb{C}^{2}$ is complex symmetric. In other words, every $2 \times 2$ matrix is unitarily equivalent to a symmetric matrix with complex entries.

In order to verify the second claim of Theorem 1, we must exhibit examples of $n$ normal operators $(n \geq 3)$ that are not complex symmetric. The following example does just this.

Example 1. We first claim that the operator $T: \mathbb{C}^{3} \rightarrow \mathbb{C}^{3}$ defined by the matrix

$$
\left(\begin{array}{lll}
0 & a & 0 \\
0 & 0 & b \\
0 & 0 & 0
\end{array}\right)
$$

(with respect to the standard basis) is a complex symmetric operator if and only if $a b=0$ or $|a|=|b|$. There are several possible cases to investigate: (i) $a=0$ or $b=0$, (ii) $|a|=|b| \neq 0$, (iii) $a \neq 0, b \neq 0$, and $|a| \neq|b|$. In particular, the final case yields 3 -normal operators that are not complex symmetric.

Case (i). If $a=0$ or $b=0$, then $T$ is the direct sum of complex symmetric operators by Corollary 1. 
Case (ii). If $|a|=|b| \neq 0$, then a short computation shows that (8) is unitarily equivalent to a constant multiple of a $3 \times 3$ nilpotent Jordan matrix. It follows from [7, Example 4] or [9, Sect. 2.2] that $T$ is a complex symmetric operator.

Case (iii). Let $a \neq 0, b \neq 0$, and $|a| \neq|b|$, and suppose toward a contradiction that $T=C T^{*} C$ for some conjugation $C$. Let $e_{1}, e_{2}, e_{3}$ denote the standard basis for $\mathbb{C}^{3}$ and observe that $e_{1}$ and $e_{3}$ span the eigenspaces of $T$ and $T^{*}$, respectively, corresponding to the eigenvalue zero. Since $T^{i} x=0$ if and only if $\left(T^{*}\right)^{i}(C x)=0$, we see that

$$
C e_{1}=\alpha_{1} e_{3}, \quad C e_{2}=\alpha_{2} e_{2}, \quad C e_{3}=\alpha_{3} e_{1},
$$

where $\alpha_{1}, \alpha_{2}, \alpha_{3}$ are certain unimodular constants. The desired contradiction will arise from computing $\left\|T e_{2}\right\|$ in two different ways. On one hand, we have

$$
\left\|T e_{2}\right\|=\left\|T^{*} C e_{2}\right\|=\left\|T^{*}\left(\alpha_{2} e_{2}\right)\right\|=\left\|T^{*} e_{2}\right\|=\|(0,0, \bar{b})\|=|b| .
$$

On the other hand, we also have $\left\|T e_{2}\right\|=\|(a, 0,0)\|=|a|$. However, this contradicts the fact that $|a| \neq|b|$. Therefore $T$ is not a complex symmetric operator.

If $n>3$, then we can use the preceding ideas to construct examples of $n$-normal operators that are not complex symmetric. Specifically, let $T: \mathbb{C}^{3} \rightarrow \mathbb{C}^{3}$ be defined as in (8), with $a b \neq 0$ and $|a| \neq|b|$ as in Case (iii). The operator $T \oplus I$ on $\mathbb{C}^{n}$, where $I$ denotes the identity operator on $\mathbb{C}^{n-3}$, is trivially $n$-normal. The same argument used in Case (iii) reveals that $T \oplus I$ is not complex symmetric.

We remark that matrices of the form (8) arose in a related unitary equivalence problem. Consideration of Jordan canonical forms reveals that each $n \times n$ matrix is similar to its transpose. On the other hand, the matrix

$$
\left(\begin{array}{lll}
0 & 2 & 0 \\
0 & 0 & 1 \\
0 & 0 & 0
\end{array}\right)
$$

is not unitarily equivalent to its transpose [14, Prob. 159].

We close this section with a corollary:

Corollary 2. If $N$ is a normal operator having spectral multiplicity $\leq 2$ and if $T$ is an operator commuting with $N$, then $T$ is a complex symmetric operator.

Proof. If $N$ is a normal operator having spectral multiplicity $\leq 2$, then we may write $N=N_{1} \oplus N_{2}^{(2)}$, where $N_{1}$ and $N_{2}$ are mutually singular $*$-cyclic normal operators [2, Thm. IX.10.20]. Moreover, we also have $T=T_{1} \oplus T_{2}$, where $T_{1}$ commutes with $N_{1}$ and $T_{2}$ commutes with $N_{2}^{(2)}$ (see the discussion following [2, Lem. IX.10.19] or [11]). From this we immediately see that $T_{1}$ is normal and $T_{2}$ is binormal [2, Cor. IX.6.9, Prop. IX.6.1.b]. It then follows from Theorem 1 that $T$ is a complex symmetric operator.

In the preceding, observe that if $N$ has spectral multiplicity two, then the conjugation corresponding to the operator $T$ depends on $T$ (as well as $N$ ).

Our next corollary asserts that any square root (normal or otherwise) of a normal operator is itself a complex symmetric operator:

Corollary 3. If $T^{2}$ is normal, then $T$ is a complex symmetric operator. 
Proof. This follows immediately from Theorem 1 and the fact that $T$ must be of the form

$$
T=A \oplus\left(\begin{array}{cc}
B & C \\
0 & -B
\end{array}\right)
$$

where $A$ and $B$ are normal and $C$ is a positive operator that commutes with $B$ [16, Thm. 1].

\section{Algebraic operators}

Definition. An operator $T \in B(\mathcal{H})$ is algebraic if $p(T)=0$ for some polynomial $p(z)$. The degree of an algebraic operator is defined to be the degree of the polynomial $p(z)$ of least degree for which $p(T)=0$.

Although the following theorem is essentially a corollary of Theorem 1, we choose to state it as a theorem since it will have several useful corollaries of its own.

Theorem 2. If $T \in B(\mathcal{H})$ is algebraic of degree $\leq 2$, then $T$ is a complex symmetric operator. This result is sharp in the sense that for each finite $n \geq 3$ and for each $\mathcal{H}$ satisfying $\operatorname{dim} \mathcal{H} \geq n$, there exists an algebraic operator on $\mathcal{H}$ of degree $n$ that is not a complex symmetric operator.

Proof. The first statement follows from Theorem 1 and an old lemma of Brown 1 , Lem. 7.1] that asserts that if $T$ is algebraic of degree $\leq 2$, then $T$ is binormal. Suppose now that $3 \leq n \leq \operatorname{dim} \mathcal{H}$ and consider the operator $T \oplus D$, where $T$ has a matrix of the form (8) with $a b \neq 0$ and $|a| \neq|b|$ and $D$ is a diagonal operator chosen so that $T \oplus D$ is algebraic of degree $n$. An argument similar to that used in Case (iii) of Example 1 shows that this operator is not complex symmetric.

Two particular classes of operators stand out for special consideration:

Corollary 4. Let $T \in B(\mathcal{H})$. If $T$ is idempotent (i.e. $T^{2}=T$ ) or nilpotent of order 2 (i.e. $\left.T^{2}=0\right)$, then $T$ is a complex symmetric operator.

A direct proof of the second portion of Corollary 4 involving the explicit construction of the associated conjugation, can be found in [5]. Yet another basic class of operators that happen to be complex symmetric are the rank-one operators:

Corollary 5. If $T \in B(\mathcal{H})$ and $\operatorname{rank}(T)=1$, then $T$ is a complex symmetric operator.

Proof. Any rank-one operator $T$ is of the form $T f=\langle f, v\rangle u$ for certain vectors $u, v$ (this operator is frequently denoted $u \otimes v$ ). Since $T^{2}-\langle u, v\rangle T=0$, it follows from Theorem 2 that $T$ is a complex symmetric operator.

It is important to note that although every operator on $\mathbb{C}^{2}$ is a complex symmetric operator (Corollary 1), there are certainly operators having rank two that are not complex symmetric operators (Example 1).

\section{Perturbations of normal operators}

In light of Corollary 5 and the fact that all normal operators are complex symmetric (see [9, Ex. 2.8] or [7, Sect. 4.1]), it is natural to attempt to identify those rank-one perturbations of normal operators that are also complex symmetric. 
Theorem 3. If $N \in B(\mathcal{H})$ is a normal operator, $U$ is a unitary operator in $\mathcal{W}^{*}(N)$ (the von Neumann algebra generated by $N$ ), $a \in \mathbb{C}$, and $v \in \mathcal{H}$, then the operator $T=N+a(U v \otimes v)$ is a complex symmetric operator.

Proof. We may without loss of generality assume that $N$ is a $*$-cyclic normal operator with cyclic vector $v$. Otherwise let $\mathcal{H}_{1}$ denote the reducing subspace of $N$ generated by $v$ and let $\mathcal{H}_{2}=\mathcal{H}_{1}^{\perp}$. Now write $N=N_{1} \oplus N_{2}$ relative to the orthogonal decomposition $\mathcal{H}=\mathcal{H}_{1} \oplus \mathcal{H}_{2}$. It follows that $N_{1}$ is $*$-cyclic and, since $\mathcal{H}_{1}$ reduces $U$, we have $T=\left(N_{1}+a\left(U_{1} v \otimes v\right)\right) \oplus N_{2}$, where $U_{1}=\left.U\right|_{\mathcal{H}_{1}}$ belongs to $\mathcal{W}^{*}\left(N_{1}\right)$.

By the Spectral Theorem, we may further presume that $N=M_{z}$ (the operator of multiplication by the independent variable on a Lebesgue space $L^{2}(\mu)$ ), that $v$ is the constant function 1 , and that $U=M_{\theta}$ (the operator of multiplication by some unimodular function $\theta$ in $L^{\infty}(\mu)$ ). At this point, a straightforward computation shows that $C f=\theta \bar{f}$ is a conjugation on $L^{2}(\mu)$ with respect to which both $M_{z}$ and $\theta \otimes 1$ are $C$-symmetric.

On an abstract level, the preceding theorem indicates that compressed shifts are complex symmetric operators. In other words, starting from the fact that the Aleksandrov-Clark unitary operators are complex symmetric, we can directly derive the fact that the compressed shift is also complex symmetric. In essence, this is the reverse of the path undertaken in [9] (which the reader may consult for further details concerning the following example).

Example 2. Let $\varphi$ denote a nonconstant inner function and let $H^{2}$ denote the Hardy space on the unit disk $\mathbb{D}$. For each $\lambda$ in the open unit disk $\mathbb{D}$, we define the unit vectors

$$
\begin{aligned}
& b_{\lambda}(z)=\frac{z-\lambda}{1-\bar{\lambda} z}, \\
& k_{\lambda}(z)=\sqrt{\frac{1-|\lambda|^{2}}{1-|\varphi(\lambda)|^{2}}} \cdot \frac{1-\overline{\varphi(\lambda)} \varphi(z)}{1-\bar{\lambda} z}, \\
& q_{\lambda}(z)=\sqrt{\frac{1-|\lambda|^{2}}{1-|\varphi(\lambda)|^{2}}} \cdot \frac{\varphi(z)-\varphi(\lambda)}{z-\lambda} .
\end{aligned}
$$

In particular, the function $k_{\lambda}$ is a normalized reproducing kernel for the so-called model space $H^{2} \ominus \varphi H^{2}$.

For each unimodular constant $\alpha$, we define the generalized Aleksandrov-Clark operator by setting

$$
U_{\lambda} f= \begin{cases}b_{\lambda} f, & f \perp q_{\lambda}, \\ \alpha k_{\lambda}, & f=q_{\lambda} .\end{cases}
$$

Each $U_{\lambda}$ is $C$-symmetric with respect to the conjugation (defined in terms of boundary functions) $[C f](z)=\overline{f z} \varphi$ on $H^{2} \ominus \varphi H^{2}$. Moreover, we also note that $q_{\lambda}=C k_{\lambda}$ for each $\lambda$.

By Theorem 3 , it follows that the operator

$$
S_{\lambda}=U_{\lambda}-(\alpha+\varphi(\lambda))\left(k_{\lambda} \otimes q_{\lambda}\right)
$$

is complex symmetric since it is of the form $U_{\lambda}+a\left(U_{\lambda} v \otimes v\right)$, where $a$ is a complex constant and $v=q_{\lambda}$. More specifically, tracing through the proof of Theorem 3 , we expect that $S_{\lambda}$ will be $C$-symmetric with respect to the $C$ described above. 
The significance of this example lies in the fact that, for the choice

$$
\alpha=-\varphi(\lambda) /|\varphi(\lambda)|
$$

the operator (12) turns out to be

$$
S_{\lambda} f=P_{\varphi}\left(b_{\lambda} f\right),
$$

the compression of the operator $M_{b_{\lambda}}: H^{2} \rightarrow H^{2}$ to the subspace $H^{2} \ominus \varphi H^{2}$. Here $P_{\varphi}$ denotes the orthogonal projection from $H^{2}$ onto $H^{2} \ominus \varphi H^{2}$. The operator $S_{0} f=P_{\varphi}(z f)$ is commonly known as the compressed shift or Jordan model operator corresponding to $\varphi$. In summary, purely operator-theoretic considerations guarantee that the operators $S_{\lambda}$ are complex symmetric. We refer the reader to [9] and [17] for more information.

In fact, the preceding example can be greatly generalized (without any reference to function theory whatsoever). Given a contraction $T \in B(\mathcal{H})$, there is a unique decomposition $\mathcal{H}=\mathcal{H}_{0} \oplus \mathcal{H}_{u}$, where $\mathcal{H}_{0}$ and $\mathcal{H}_{u}$ are both $T$-invariant, $\left.T\right|_{\mathcal{H}_{u}}$ is unitary, and $\left.T\right|_{\mathcal{H}_{0}}$ is completely nonunitary (i.e., $\left.T\right|_{\mathcal{H}_{0}}$ is not unitary when restricted to any of its invariant subspaces). The operator $D_{T}=\left(I-T^{*} T\right)^{1 / 2}$ is called the defect operator of $T$, and the defect spaces of $T$ are defined to be the subspaces $\mathcal{D}_{T}=\overline{D_{T} \mathcal{H}}$ and $\mathcal{D}_{T^{*}}=\overline{D_{T^{*}} \mathcal{H}}$. The defect indices of $T$ are the numbers $\partial_{T}=$ $\operatorname{dim} \mathcal{D}_{T}$ and $\partial_{T^{*}}=\operatorname{dim} \mathcal{D}_{T^{*}}$. We say that $T \in C_{0}$. if $T^{n} \rightarrow 0$ (SOT) and that $T \in C_{.0}$ if $T^{*} \in C_{0}$. Finally, we also define $C_{00}=C_{0} . \cap C_{.0}$.

It turns out that any Hilbert space contraction with defect indices $\partial_{T}=\partial_{T^{*}}=1$ is complex symmetric. Although this is known (see [3, Cor. 3.2] for a general proof) and easy to prove if $T \in C_{00}$ (see [9, Thm. 5.1], 7, Prop. 3], and [17, Lem. 2.1]), we are able to establish this result in the abstract - without the use of characteristic functions and complex analysis.

Corollary 6. If $T \in B(\mathcal{H})$ is a contraction such that $\partial_{T}=\partial_{T^{*}}=1$, then $T$ is a complex symmetric operator.

Proof. Since $\partial_{T}=1$, it follows that $I-T^{*} T=u \otimes u$ for some nonzero vector $u$. If $x$ is any vector orthogonal to $u$, then we have

$$
\|x\|^{2}-\|T x\|^{2}=\left\langle\left(I-T^{*} T\right) x, x\right\rangle=|\langle u, x\rangle|^{2}=0 .
$$

Thus $T$ is isometric on a subspace of $\mathcal{H}$ having codimension one. Similarly, we see that $I-T T^{*}$ is also of rank one, whence $I-T T^{*}=v \otimes v$ for some nonzero vector $v$. Putting this together, we find that $T=\left.T\right|_{u^{\perp}}+c(u \otimes v)$ for some constant $c$. In particular, there exists a unitary $U$ such that $T=U+c^{\prime}(u \otimes v)$ is a rank-one perturbation of $U$. Since $T$ is of the form $T=U+a(U v \otimes v)$ where $U$ is unitary, it follows from Theorem 3 that $T$ is a complex symmetric operator.

Following Theorem 3 in another direction, we obtain the following:

Corollary 7. Let $T=A+i B$ denote the Cartesian decomposition of $T \in B(\mathcal{H})$ (i.e. $A=A^{*}$ and $B=B^{*}$ ). If $\operatorname{rank}(A)=1$ or $\operatorname{rank}(B)=1$, then $T$ is a complex symmetric operator. 
Proof. If $A$ has rank one, then $A=a(v \otimes v)$ for some $a \in \mathbb{R}$ and $v \in \mathcal{H}$. Apply Theorem 3 with $N=i B$ and $U=I$.

The preceding corollary easily furnishes many examples of nonnormal complex symmetric operators. Indeed, if $A$ is an arbitrary selfadjoint operator and $B$ is a rank-one selfadjoint operator that does not commute with $A$, then $T=A+i B$ is a nonnormal complex symmetric operator. Despite the apparent simplicity of such a recipe, nontrivial examples abound. Consider the following example:

Example 3. It is well known that the Volterra integration operator

$$
[V f](x)=\int_{0}^{x} f(y) d y
$$

on $L^{2}[0,1]$ is a rank-one selfadjoint perturbation of a skew-selfadjoint operator (see [4] or [13, Pr. 188] for further details). Indeed, a short computation shows that the selfadjoint component of $V$ is

$$
A=\frac{1}{2}\left(V+V^{*}\right)=\int_{0}^{1} f(y) d y=\frac{1}{2}(1 \otimes 1)
$$

where the 1 above denotes the constant function. By Corollary 7 we conclude that $V$ is a complex symmetric operator. In fact, $V=C V^{*} C$, where $C$ denotes the conjugation $[C f](x)=\overline{f(1-x)}$ on $L^{2}[0,1]$ (see [8, Ex. 6] and [9, Sect. 4.1]).

Setting $U=I$ in Theorem 3 provides a generalization of Corollary 7

Corollary 8. If $N \in B(\mathcal{H})$ is a normal operator, $P$ is a rank-one orthogonal projection, and $a \in \mathbb{C}$, then $T=N+a P$ is a complex symmetric operator.

It is important to note that not every rank-one perturbation of a normal operator will be complex symmetric (unless $\operatorname{dim} \mathcal{H}=2$; see Corollary 1). In fact, even a rankone perturbation of an orthogonal projection may fail to be complex symmetric:

Example 4. We claim that the operator $T: \mathbb{C}^{3} \rightarrow \mathbb{C}^{3}$ defined by the matrix

$$
\left(\begin{array}{lll}
0 & 0 & 1 \\
0 & 1 & 1 \\
0 & 0 & 0
\end{array}\right)
$$

(with respect to the standard basis) is not a complex symmetric operator. First observe that the eigenspaces of $T$ (and hence of $T^{*}$ ) for the eigenvalues 0 and 1 are both one-dimensional. The eigenspaces of $T$ corresponding to the eigenvalues 0 and 1 are spanned by the unit vectors $v_{0}=(1,0,0)$ and $v_{1}=(0,1,0)$, respectively. The eigenspaces of $T^{*}$ corresponding to the eigenvalues 0 and 1 are spanned by the unit vectors $w_{0}=(0,0,1)$ and $w_{1}=\left(0, \frac{1}{\sqrt{2}}, \frac{1}{\sqrt{2}}\right)$, respectively. If $C$ is a conjugation such that $T=C T^{*} C$, then $0=\left|\left\langle v_{0}, v_{1}\right\rangle\right|=\left|\left\langle C v_{1}, C v_{0}\right\rangle\right|=\left|\left\langle w_{1}, w_{0}\right\rangle\right|=\frac{1}{\sqrt{2}}$, which is absurd.

\section{Partial isometries}

In this section, we attempt to classify those partial isometries that are complex symmetric. This question is related to the preceding material in the sense that if $\varphi(0)=0$ in Example 2, then the corresponding compressed shift operator is a complex symmetric partial isometry. 
Given only the dimensions of the kernels of a partial isometry and its adjoint, the following theorem is as definitive as possible:

Theorem 4. Let $T \in B(\mathcal{H})$ be a partial isometry.

(i) If $\operatorname{dim} \operatorname{ker} T=\operatorname{dim} \operatorname{ker} T^{*} \leq 1$, then $T$ is a complex symmetric operator.

(ii) If $\operatorname{dim} \operatorname{ker} T \neq \operatorname{dim} \operatorname{ker} T^{*}$, then $T$ is not a complex symmetric operator.

(iii) If $2 \leq \operatorname{dim} \operatorname{ker} T=\operatorname{dim} \operatorname{ker} T^{*} \leq \infty$, then either possibility can (and does) occur.

Proof. (i) If $\operatorname{dim} \operatorname{ker} T=\operatorname{dim} \operatorname{ker} T^{*}=0$, then $T^{*} T=T T^{*}=I$, whence $T$ is unitary and hence complex symmetric. Suppose that $T$ is a partial isometry satisfying $\operatorname{dim} \operatorname{ker} T=\operatorname{dim} \operatorname{ker} T^{*}=1$ and that $\operatorname{ker} T$ and $\operatorname{ker} T^{*}$ are spanned by the unit vectors $v$ and $w$, respectively. Since the operator $N=T+w \otimes v$ is unitary, it follows that $T=N-N v \otimes v$ is a complex symmetric operator by Theorem 3 . For a different proof, see [3, Cor. 3.2].

(ii) We prove the contrapositive. If $T$ is $C$-symmetric, then it is easy to see that $T x=0$ if and only if $T^{*}(C x)=0$. Therefore $C$ furnishes an isometric, conjugatelinear bijection between $\operatorname{ker} T$ and $\operatorname{ker} T^{*}$, whence $\operatorname{dim} \operatorname{ker} T=\operatorname{dim} \operatorname{ker} T^{*}$.

(iii) This portion of the theorem follows upon consideration of several examples. It is trivial to produce complex symmetric partial isometries with $\operatorname{dim} \operatorname{ker} T=$ $\operatorname{dim} \operatorname{ker} T^{*}=n$ for any $n$. In fact, $T=I \oplus 0$, where 0 is the zero operator on an $n$-dimensional Hilbert space, is such an example. On the other hand, finding partial isometries that are not complex symmetric when $2 \leq n \leq \infty$ is more involved.

For the remainder of this proof, we choose not to distinguish between matrices and the operators they induce (with respect to the standard basis). We must first study a certain auxiliary matrix that will be used in our construction. Specifically, we intend to prove that

$$
A=\left(\begin{array}{ccc}
0 & \frac{1}{2} & 0 \\
0 & 0 & \frac{1}{4} \\
1 & 0 & 0
\end{array}\right)
$$

is not a complex symmetric operator. This will follow from a careful study of the eigenstructures of $A$ and $A^{*}$. First, note that the eigenvalues of $A$ are

$$
\lambda_{1}=\frac{1}{2}, \quad \lambda_{2}=-\frac{1}{4}+i \frac{\sqrt{3}}{4}, \quad \lambda_{3}=-\frac{1}{4}-i \frac{\sqrt{3}}{4},
$$

and that these are also the eigenvalues of $A^{*}$. A straightforward computation shows that corresponding unit eigenvectors of $A$ are

$$
\begin{aligned}
& v_{1}=\frac{1}{\sqrt{6}}(1,1,2), \\
& v_{2}=\frac{1}{2 \sqrt{6}}(-1+i \sqrt{3},-1-i \sqrt{3}, 4), \\
& v_{3}=\frac{1}{2 \sqrt{6}}(1+i \sqrt{3}, 1-i \sqrt{3},-4) .
\end{aligned}
$$

Since $A$ has three distinct eigenvalues, it follows that $v_{1}, v_{2}, v_{3}$ must be sent to unimodular scalar multiples of the corresponding unit eigenvectors

$$
\begin{aligned}
& w_{1}=\frac{1}{3}(2,2,1), \\
& w_{2}=\frac{1}{3}(-1-i \sqrt{3},-1+i \sqrt{3}, 1), \\
& w_{3}=\frac{1}{3}(-1+i \sqrt{3},-1-i \sqrt{3}, 1)
\end{aligned}
$$


of $A^{*}$. Now observe that

$$
\left|\left\langle v_{1}, v_{2}\right\rangle\right|=\left|\left\langle v_{2}, v_{3}\right\rangle\right|=\left|\left\langle v_{3}, v_{1}\right\rangle\right|=\frac{1}{2},
$$

whereas

$$
\left|\left\langle w_{1}, w_{2}\right\rangle\right|=\left|\left\langle w_{2}, w_{3}\right\rangle\right|=\left|\left\langle w_{3}, w_{1}\right\rangle\right|=\frac{1}{3} .
$$

The same argument used in Example 4 now reveals that $A$ cannot be a complex symmetric operator.

We are now ready to construct our desired partial isometry. Noting that

$$
A^{*} A=\left(\begin{array}{ccc}
1 & 0 & 0 \\
0 & \frac{1}{4} & 0 \\
0 & 0 & \frac{1}{16}
\end{array}\right),
$$

we see that if $2 \leq n \leq \infty$, then the $n \times 3$ matrix

$$
B=\left(\begin{array}{ccc}
0 & \frac{\sqrt{3}}{2} & 0 \\
0 & 0 & \frac{\sqrt{15}}{4} \\
0 & 0 & 0 \\
\vdots & \vdots & \vdots \\
0 & 0 & 0
\end{array}\right)
$$

satisfies $A^{*} A+B^{*} B=I$ (the $3 \times 3$ identity matrix). The $(n+3) \times(n+3)$ matrix

$$
T=\left(\begin{array}{ll}
A & 0 \\
B & 0
\end{array}\right)
$$

is a partial isometry since $T^{*} T$ is the orthogonal projection

$$
P=\left(\begin{array}{ll}
I & 0 \\
0 & 0
\end{array}\right) .
$$

Since it is clear from the construction of $T$ that $\operatorname{dim} \operatorname{ker} T=\operatorname{dim} \operatorname{ker} T^{*}=n$, we need only prove that $T$ is not a complex symmetric operator.

Suppose toward a contradiction that $T$ is $C$-symmetric. By [8, Thm. $2 \&$ Cor. 1], we may write $T=C J P$, where $J$ is an auxiliary conjugation that commutes with $P$. Since $J P=P J$ we find that

$$
J(P T) J=J(P C J P) J=P J C P=T^{*} P=(P T)^{*},
$$

whence $P T$ is $J$-symmetric. However,

$$
P T=\left(\begin{array}{cc}
A & 0 \\
0 & 0
\end{array}\right)
$$

and the same argument that showed that $A$ was not a complex symmetric operator also shows that $P T$ is not a complex symmetric operator. This contradiction shows that our partial isometry $T$ is not a complex symmetric operator, as desired.

We remark that in the final paragraph of the proof, we could have appealed to the fact that the Aluthge transform of a complex symmetric operator is also complex symmetric [5].

Based upon the preceding material, we can prove that every partial isometry on a three-dimensional Hilbert space is complex symmetric:

Corollary 9. If $\operatorname{dim} \mathcal{H}=3$, then every partial isometry $T \in B(\mathcal{H})$ is complex symmetric. 
Proof. Suppose that $\operatorname{dim} \mathcal{H}=3$ and that $T$ is a partial isometry on $\mathcal{H}$. There are four cases to discuss:

(i) If $\operatorname{dim} \operatorname{ker} T=0$, then $T$ is unitary and thus complex symmetric. Indeed, the Spectral Theorem asserts that $T$ has a diagonal matrix representation with respect to some orthonormal basis of $\mathcal{H}$.

(ii) If $\operatorname{dim} \operatorname{ker} T=1$, then $T$ is complex symmetric by (i) of Theorem 4 , The condition $\operatorname{dim} \operatorname{ker} T=\operatorname{dim} \operatorname{ker} T^{*}$ holds trivially since $\mathcal{H}$ is finitedimensional.

(iii) If $\operatorname{dim} \operatorname{ker} T=2$, then $\operatorname{rank}(T)=1$. By Corollary [5 it follows that $T$ is complex symmetric.

(iv) If $\operatorname{dim} \operatorname{ker} T=3$, then $T=0$ and the result is trivial.

Based on the construction used in the proof of Theorem 4, it is clear that many partial isometries that are not complex symmetric exist if the dimension of the underlying Hilbert space is $\geq 5$. On the other hand, we were for a considerable time unable to determine whether all partial isometries on a four-dimensional Hilbert space are complex symmetric (they are). In this setting, the method of Corollary 9 suffices to resolve all but the case $\operatorname{dim} \operatorname{ker} T=2$.

Significant numerical evidence in favor of the assertion that all partial isometries on a four-dimensional Hilbert space are complex symmetric has been produced by J. Tener [18. We refer the reader to [10] for the resolution of this problem.

\section{REFERENCES}

[1] Brown, A., The unitary equivalence of binormal operators, Amer. J. Math. 76 (1954), 414434. MR0062355 (15:967e)

[2] Conway, J.B., A Course in Functional Analysis (second edition), Graduate Texts in Mathematics, 96, Springer-Verlag, New York, 1990. MR1070713 (91e:46001)

[3] Chevrot, N., Fricain, E., Timotin, D., The characteristic function of a complex symmetric contraction, Proc. Amer. Math. Soc. 135 (2007), 2877-2886. MR2317964 (2008c:47025)

[4] Garcia, S.R., Approximate antilinear eigenvalue problems and related inequalities, Proc. Amer. Math. Soc. 136 (2008), no. 1, 171-179 . MR2350402(2008k:47022)

[5] Garcia, S.R., Aluthge transforms of complex symmetric operators, Integral Equations Operator Theory 60 (2008), no. 3, 357-367. MR2392831 (2008m:47052)

[6] Garcia, S.R., Means of unitaries, conjugations, and the Friedrichs operator, J. Math. Anal. Appl. 335 (2007), 941-947. MR2345511 (2008i:47070)

[7] Garcia, S.R., Putinar, M., Complex symmetric operators and applications, Trans. Amer. Math. Soc. 358 (2006), 1285-1315. MR.2187654 (2006j:47036)

[8] Garcia, S.R., Putinar, M., Complex symmetric operators and applications II, Trans. Amer. Math. Soc. 359 (2007), 3913-3931. MR2302518 (2008b:47005)

[9] Garcia, S.R., Conjugation and Clark Operators, Contemp. Math. 393 (2006), 67-112. MR2198373 (2007b:47073)

[10] Garcia, S.R., Wogen, W.R., Complex symmetric partial isometries, J. Funct. Anal. 257 (2009), no. 4, 1251-1260. MR.2535469

[11] Gilbreath, T.M., Wogen, W.R., Remarks on the structure of complex symmetric operators, Integral Equations Operator Theory 59 (2007), no. 4, 585-590. MR2370050

[12] Hadwin, D., Laurie, C., Reflexive binormal operators, J. Funct. Anal. 123 (1994), no. 1, 99-108. MR 1279297 (96c:47049)

[13] Halmos, P.R., A Hilbert Space Problem Book (Second Edition), Springer-Verlag, New York, 1982. MR675952(84e:47001)

[14] Halmos, P.R., A Linear Algebra Problem Book, The Dolciani Mathematical Expositions, 16, Mathematical Association of America, Washington, DC, 1995. MR1310775 (96e:15001) 
[15] Radjavi, H., Rosenthal, P., Invariant Subspaces, Ergebnisse der Mathematik und ihrer Grenzgebiete, Band 77, Springer-Verlag, New York-Heidelberg, 1973. MR.0367682 (51:3924)

[16] Radjavi, H., Rosenthal, P., On roots of normal operators, J. Math. Anal. Appl. 34 (1971), 653-664. MR0278097 (43:3829)

[17] Sarason, D., Algebraic properties of truncated Toeplitz operators, Oper. Matrices 1 (2007), no. 4, 491-526. MR2363975 (2008i:47060)

[18] Tener, J.E., Unitary equivalence to a complex symmetric matrix: An algorithm, J. Math. Anal. Appl. 341 (2008), no. 1, 640-648. MR2394112 (2008m:15062)

Department of Mathematics, Pomona College, Claremont, California 91711

E-mail address: Stephan.Garcia@pomona.edu

$U R L:$ http://pages.pomona.edu/ ${ }^{\text {sg064747 }}$

Department of Mathematics, CB \#3250, Phillips Hall, University of North Carolina

at Chapel Hill, Chapel Hill, North Carolina 27599

E-mail address: wrw@email.unc.edu

$U R L:$ http://www.math.unc.edu/Faculty/wrw 